Bull. Austral. Math. Soc.

VoL. 40 (1989) [337-344]

\title{
ALMOST COMPLEX STRUCTURES ON THE ORTHOGONAL TWISTOR BUNDLE
}

\author{
KICHOON YANG
}

\begin{abstract}
We give a construction of $2^{s}, s=n(n-1) / 2$, many natural almost complex structures on the orthogonal $t$ wistor bundle over a $2 n$-dimensional Riemannian manifold. The usual almost complex structures are then characterised by the condition that they correspond to integrable invariant complex structures on the standard fibre which is identified with the hermitian symmetric space $S O(2 n) / U(n)$.
\end{abstract}

\section{O. INTRODUC'TION}

Recently, much attention has been paid to the investigation of the (orthogonal) twistor bundle, $\mathcal{T} \rightarrow N$, over an evell-dimensional Riemannian manifold $N$ and its application to the theory of harmonic maps. See, for example, $[2,3]$ and many references cited therein. A crucial aspect of the twistor method is the construction of the two almost complex structures, denoted by $J_{+}$and $J_{-}\left([3]\right.$ calls them $J_{1}$ and $\left.J_{2}\right)$, on the total space of the twistor bundle. $J_{-}$, which is never integrable, plays an important rôle in the study of conformal minimal immersions from a Riemann surface $M$ into $N$. When the dimension of the target manifold $N$ happens to be four, the twistor method is particularly effective and [3] proved the following result.

A conformal immersion $f: M \rightarrow N$ is minimal if and only if its twistor lift $T_{f}: M \rightarrow T$ is $J_{-}$-complex. (The twistor lift of an immersion $f: M \rightarrow N$ is obtained by composing the Gauss map $M \rightarrow G_{2}(T N)$, the Grassmanm bundle of oriented tangent two-planes of $N$, with the projection $G_{2}(T N) \rightarrow \mathcal{T}$.)

In this paper we give a systematic account of the construction of almost complex structures on $\mathcal{T}$. In particular, we show that there are not two but $2^{\gamma}$, with $\gamma=$ $n(n-1) / 2(\operatorname{dim} N=2 n)$, many natural almost complex structures on $\mathcal{T}$ (Section 2 (16)). With the possible exception of $J_{+}$none of these almost complex structures are ever integrable. However, these structures are still quite useful in manufacturing new harmonic maps from a Riemann surface into $N$.

Received 1 November, 1988

The author would like to thank Simon Salamon for a stimulating conversation and Gary Jensen for making available the preprint [4].

Copyright Clearance Centre, Inc. Serial-fee code: 0004-9729/89 \$A2.00+0.00. 


\section{The Hermetian Symmetric Space $S O(2 n) / U(n)$}

Let $i: U(n) \rightarrow S O(2 n)$ be the Lie group monomorphism induced by the identifcation

$$
\mathbf{R}^{2 n} \leftrightarrow \mathrm{C}^{n}, \quad\left(x^{\alpha}\right) \leftrightarrow\left(x^{1}+i x^{2}, \ldots, x^{2 n-1}+i x^{2 n}\right)
$$

We then have

$$
i(U(n))=\left\{X \in S O(2 n):{ }^{t} X J_{n} X=J_{n}\right\}
$$

where $J_{n}=J_{1} \oplus \ldots \oplus J_{1}=\left[\begin{array}{ccc}J_{1} & & \\ & \ddots & \\ & & J_{1}\end{array}\right], J_{1}=\left[\begin{array}{cc}0 & -1 \\ 1 & 0\end{array}\right]$.

Convention. We will identify $U(n)$ with $i(U(n))$ and write $U(n)$ instead of $i(U(n))$, $u(n)$ instead of $i_{\star_{e}}(u(n))$, and so on.

At the Lie algebra level we have

$$
i_{\star_{e}}(\mathbf{u}(n))=\mathbf{u}(n)=\left\{X \in \mathbf{o}(2 n):{ }^{t} X J_{n}+J_{n} X=0\right\}
$$

Let $T$ be a maximal torus in $S O(2 n)$ given by

$$
T=\left\{\operatorname{diag}\left(D_{1}, \ldots, D_{n}\right): D_{i}=\left(\begin{array}{cc}
c_{i} & -s_{i} \\
s_{i} & c_{i}
\end{array}\right), c_{i}=\cos 2 \pi x_{i}, s_{i}=\sin 2 \pi x_{i}\right\}=S O(2)^{n}
$$

Notation. We identify $S O(2)$ with $U(1)$ and write $e^{i x}$ (or $\exp (i x)$ ) instead of $\left(\begin{array}{cc}\cos x & -\sin x \\ \sin x & \cos x\end{array}\right)$.

We have the Lie algebra composition

$$
\mathbf{o}(2 n)=\mathbf{t} \oplus \mathbf{m}
$$

where $\mathbf{t}$ denotes the Lie algebra of $T$ and $\mathbf{m}$ is the orthogonal complement to $\mathbf{t}$ relative to the Killing form.

Let $E_{i j}=\left[e_{m n}\right], F_{i j}=\left[f_{m n}\right], E_{i j}^{\prime}=\left[e_{m n}^{\prime}\right]$ and $F_{i j}^{\prime}=\left[f_{m n}^{\prime}\right]$ be $2 n \times 2 n$ matrices with all entries zero except for

$$
\begin{aligned}
& e_{2 i-1,2 j-1}=e_{2 i, 2 j}=-e_{2 j-1,2 i-1}=-e_{2 j, 2 i}=1 \\
& -f_{2 i-1,2 j}=f_{2 i, 2 j-1}=-f_{2 j-1,2 i}=f_{2 j, 2 i-1}=1 \\
& e_{2 i-1,2 j-1}^{\prime}=-e_{2 i, 2 j}^{\prime}=-e_{2 j-1,2 i-1}^{\prime}=e_{2 j, 2 i}^{\prime}=1 \\
& f_{2 i-1,2 j}^{\prime}=f_{2 i, 2 j-1}^{\prime}=-f_{2 j-1,2 i}^{\prime}=-f_{2 j, 2 i-1}^{\prime}=1
\end{aligned}
$$


Then

$$
\mathbf{m}=\sum V_{i j} \oplus V_{i j}^{\prime} \quad(1 \leqslant i<j \leqslant n),
$$

where $V_{i j}=\mathbf{R}-\operatorname{span}\left\{E_{i j}, F_{i j}\right\}$ and $V_{i j}^{\prime}=\mathbf{R}-\operatorname{span}\left\{E_{i j}^{\prime}, E_{i j}^{\prime}\right\}$.

For $t=\operatorname{diag}\left(D_{1}, \ldots, D_{n}\right) \in T, v=x E_{i j}+y F_{i j} \in V_{i j}$, and $v^{\prime}=x E_{i j}^{\prime}+y F_{i j}^{\prime} \in V_{i j}^{\prime}$ we compute that

$$
\begin{gathered}
A d_{t}: v \leftrightarrow x+i y \mapsto \exp \left(2 \pi i\left(x_{i}-x_{j}\right)\right) \cdot(x+i y), \\
v^{\prime} \leftrightarrow x+i y \mapsto \exp \left(2 \pi i\left(x_{i}+x_{j}\right)\right) \cdot(x+i y),
\end{gathered}
$$

where we use the complex notation to write $v, v^{\prime}$ relative to their respective bases. (5) means that the root spaces of $S O(2 n)$ relative to $T$ are $V_{i j}$ and $V_{i j}^{\prime}$ and the corresponding roots are

$$
\Delta=\left\{ \pm\left(x_{i}-x_{j}\right), \pm\left(x_{i}+x_{j}\right): 1<i<j \leqslant n\right\} .
$$

A routine computation then reveals that

$$
\mathbf{u}(n)=\mathbf{t} \oplus \sum V_{i j} .
$$

It follows that

$$
\mathbf{n}=\oplus \sum V_{i j}^{\prime}
$$

is the orthogonal complement to $u(n)$ in $o(2 n)$ relative to the Killing form.

Via $\pi_{\star_{e}}(\pi: S O(2 n) \rightarrow S O(2 n) / U(n)) n$ is identified with the tangent space at the identity coset of $S O(2 n) / U(n)$.

$$
\mathbf{n} \leftrightarrow T_{0}(S O(2 n) / U(n)), \quad 0=U(n) .
$$

Let $\Omega=\left(\Omega_{\beta}^{\alpha}\right), 1 \leqslant \alpha, \beta \leqslant 2 n$, denote the Maurer-Cartan form of $S O(2 n)$. The decompositions in $(3,6,7)$ induce the decompositions

$$
\Omega=\Omega_{\mathrm{u}(\mathrm{n})}+\Omega_{\mathrm{n}}, \quad \Omega_{\mathrm{n}}=\sum \Omega_{V_{i j}^{\prime}}
$$

We compute that

$$
\Omega_{V_{i j}^{\prime}}=\frac{1}{2}\left[\left(\Omega_{2 j-1}^{2 i-1}-\Omega_{2 j}^{2 i}\right) \otimes E_{i j}^{\prime}+\left(\Omega_{2 j-1}^{2 i}+\Omega_{2 j}^{2 i-1}\right) \otimes F_{i j}^{\prime}\right], \text { (no sum). }
$$

Put

$$
\Theta^{\prime i j}=\frac{1}{2}\left[\left(\Omega_{2 j-1}^{2 i-1}-\Omega_{2 j}^{2 i}\right)+\epsilon_{i j} i\left(\Omega_{2 j-1}^{2 i}+\Omega_{2 j}^{2 i-1}\right)\right], \text { (no sum) }
$$

where $\varepsilon_{i j}= \pm 1$ (but fixed). 
Proposition. $S O(2 n) / U(n)$ posseses exactly $2^{\gamma}, \gamma=n(n-1) / 2$, many invariant almost complex structures.

Proof: See [1, p.501] or [8, Chapter II, Section 9].

The invariant almost complex structures on $S O(2 n) / U(n)$ can be described as follows: Choose $\left\{\varepsilon_{i j}=+1\right.$ or $\left.-1: 1 \leqslant i<j \leqslant n\right\}$ and let the pullbacks (by a local section of $S O(2 n) \rightarrow S O(2 n) / U(n))$ of $\left(\Theta^{\prime i j}\right)$ span type $(1,0)$ forms on $S O(2 n) / U(n)$. The invariant almost complex structure on $S O(2 n) / U(n)$ corresponding to the choice $\left\{\varepsilon_{i j}\right\}$ will be denoted by

$$
\oplus \sum \varepsilon_{i j} J_{1}, \quad 1 \leqslant i<j \leqslant n .
$$

Letting $\varepsilon_{i j}=1$ for every $i$ and $j$ we obtain an integrable invariant almost complex structure. $S O(2 n) / U(n)$ has exactly one other integrable invariant almost complex structure which is the conjugate structure, corresponding to the choice $\varepsilon_{i j}=-1$ for every $i$ and $j$.

Any invariant metric on $S O(2 n) / U(n)$ is given by the pullback of the symmetric product

$$
c \cdot \sum \Theta^{\prime i j} \cdot \bar{\Theta}^{\prime i j}, \quad c>0 .
$$

Let $\langle$,$\rangle denote the standard inner product in \mathbf{R}^{2 n}$ and put

$$
\mathcal{F}=\left\{J \in \operatorname{Aut}^{+}\left(\mathrm{R}^{2 n}\right): J^{2}=-I, \quad\langle v, w\rangle=\langle J v, J w\rangle\right\},
$$

where $\mathrm{Aut}^{+}\left(\mathbf{R}^{2 n}\right)$ denotes the set of orientation preserving automorphisms of $\mathbf{R}^{2 n} \cdot \mathcal{F}$ is the set of all orientation preserving orthogonal complex structures of the vector space $\mathbf{R}^{2 n}$.

Let $A=\left(A_{\alpha}\right)=\left(A_{1}, \ldots, A_{2 n}\right) \in S O(2 n)$ and consider the assignment

$$
A \mapsto J_{A} \in \mathcal{F}
$$

given by the formula

$$
J_{A}\left(A_{2 i-1}\right)=A_{2 i} \text { and } J_{A}\left(A_{2 i}\right)=-A_{2 i-1}, \quad 1 \leqslant i \leqslant n ;
$$

that is, the malrix of $J_{A}$ relative to the basis $\left(A_{\alpha}\right)$ is just $J_{n}$. This assignment induces a bijection

$$
S O(2 n) / U(n) \stackrel{\cong}{F}
$$

as it is easily seen that $J_{A}=J_{B}$ if $B=A \cdot U$ for some $U \in U(n)$. 


\section{THE ORTIIOGONAL TWISTOR BUNDLE}

Let $N$ denote a connected oriented $2 n$-dimensional Riemannian manifold and also let

$$
\pi: S O(N) \rightarrow N
$$

denote the $S O(2)$-principal bundle of oriented orthonormal frames over $N$.

The $\mathbf{R}^{2 n}$-valued canonical form, denoted by $\Theta=\left(\Theta^{\alpha}\right)$, on $S O(N)$ is given by

$$
\Theta(X)=e^{-1}\left(\pi_{\star} X\right), \quad X \in T_{(x, e)} S O(N)
$$

where $e$ is identified with a linear map $\mathbf{R}^{2 n} \rightarrow T_{x} N$.

We have

$$
d \Theta^{\alpha}=-\Omega_{\beta}^{\alpha} \wedge \Theta^{\beta}
$$

where $\Omega=\left(\Omega_{\beta}^{\alpha}\right), 1 \leqslant \alpha, \beta \leqslant 2 n$, is the $o(2 n)$-valued Levi-Civita connection form on $S O(N)$.

Definition. Put $\mathcal{T}=\{(x, J): x \in N, J$ is an orientation preserving orthogonal complex structure of the vector space $\left.T_{x} N\right\} . \pi_{2}: \mathcal{T} \rightarrow N,(x, J) \mapsto x$, is called the orthogonal twistor bundle over $N$. ( $T \rightarrow N$ depends only on the conformal structure of $N$.)

Consider the projection

$$
\pi_{1}: S O(N) \rightarrow \mathcal{T}, \quad\left(x ; e_{1}, \ldots, e_{2 n}\right) \mapsto\left(x ; J_{e}\right),
$$

where $J_{e}: e_{2 i-1} \mapsto e_{2 i}, e_{2 i} \mapsto e_{2 i-1}$

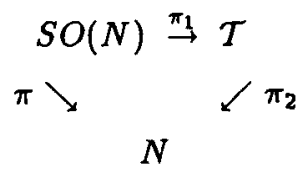

Take $x \in N$ and fix an oriented orthonormal basis $\delta=\left(\delta_{1}, \ldots, \delta_{2 n}\right)$ of $T_{x} N$. For any $e=\left(e_{\alpha}\right) \in S O(N)_{x}=\pi^{-1}(x)$ we write $e_{\alpha}=e_{\alpha}^{\beta} \delta_{\beta}$ and obtain the identification

$$
S O(N)_{x} \cong S O(2 n), \quad e \mapsto\left(e_{\alpha}^{\beta}\right)
$$

Likewise the isomorphism $\delta: T_{x} N \rightarrow \mathbf{R}^{2 n}, \delta_{\alpha} \mapsto\left(\partial / \partial x^{\alpha}\right)$, induces an identification

$$
\mathcal{T}_{\mathbf{x}} \cong \mathcal{F}=S O(2 n) / U(n)
$$


Sumning up the preceding discussion we have

$$
\mathcal{T}=S O(N) \times_{S O(2 n)} S O(2 n) / U(n)=S O(N) U(n),
$$

and $\pi_{1}: S O(N) \rightarrow \mathcal{T}$ is a principal $U(n)$-fibration.

Recall from Section 1 the Lie algebra decomposition

$$
\mathbf{o}(2 n)=\mathbf{u}(n) \oplus \mathbf{n}, \quad \mathbf{n}=\oplus \sum V_{i j}^{\prime} \quad(1 \leqslant i<j \leqslant n) .
$$

The o(2n)-valued connection form $\Omega$ on $S O(N)$ decomposes accordingly,

$$
\Omega=\Omega_{\mathbf{u}(n)} \oplus \Omega_{\mathbf{n}}
$$

So,

$$
\Omega_{\mathbf{n}}=\sum \Omega_{V_{i j}^{\prime}} \quad(1 \leqslant i<j \leqslant n),
$$

where

$$
\Omega_{V_{i j}^{\prime}}=\frac{1}{2}\left[\left(\Omega_{2 j-1}^{2 i-1}-\Omega_{2 j}^{2 i}\right) \otimes E_{i j}^{\prime}+\left(\Omega_{2 j-1}^{2 i}+\Omega_{2 j}^{2 i-1}\right) \otimes F_{i j}^{\prime}\right], \text { (no sum) }
$$

P'ut

$$
\Theta^{\prime i j}=\frac{1}{2}\left[\left(\Omega_{2 j-1}^{2 i-1}-\Omega_{2 j}^{2 i}\right)+\varepsilon_{i j} i\left(\Omega_{2 j-1}^{2 i}+\Omega_{2 j}^{2 i-1}\right)\right] .
$$

Define a symmetric bilinear form on $S O(N)$ by

$$
B={ }^{t} \Theta \cdot \Theta+c \cdot \sum \Theta^{\prime i j} \cdot \bar{\Theta}^{\prime i j}, \quad c>0 .
$$

We then easily verify that: (i) $R_{g}^{\star} B=B$, where $g \in U(n)$ and $R_{g}$ denotes the right multiplication by $g$ on $S O(N)$; (ii) $B(v, w)=0$ if one of $v, w$ is a vertical vector of the fibration $S O(N) \rightarrow \mathcal{T}$. Consequently, there exists a unique Riemannian metric, denoted by $d s_{T}^{2}$ on $\mathcal{T}$ such that

$$
\pi_{1}^{\star} d s_{T}^{2}=B
$$

Remark. $(S O(N), B) \rightarrow\left(T, d s_{T}^{2}\right)$ is a Riemannian submersion with totally geodesic fibres.

Let $(x, J) \in \mathcal{T}$ and $V_{(x, J)}$ denote the subspace of $T_{(x, J)} \mathcal{T}$ tangential to the fibre $\pi_{2}^{-1}(x)$. We also let $H_{(x, J)} \subset T_{(x, J)} \mathcal{T}$ denote the orthogonal complement to $V_{(x, J)}$ with 
respect to the metric $d s_{T}^{2}$. $\left(H_{(x, J)}\right.$ is well-defined independent of the choice of $c$ in (12).)

$$
T_{(x, J)} \mathcal{T}=H_{(x, J)} \oplus V_{(x, J)}
$$

The distribution defined by $H_{(x, J)},(x, J) \in \mathcal{T}$, will be called the horizontal distribution of $\mathcal{T} \rightarrow N$.

$\pi_{2^{\star}}$ gives an isomorphism $H_{(x, J)} \stackrel{\cong}{\rightarrow} T_{x} N$. Now $J$ is a complex structure on $T_{x} N$, hence via the above isomorphism $J$ defines a complex structure on $H_{(x, j)}$. On the other hand, $\pi_{2}^{-1}(x) \stackrel{\cong}{\rightrightarrows} S O(2 n) / U(n)$ and an invariant almost complex structure on $S O(2 n) / U(n)$ gives rise to a complex structure on $V_{(x, J)}$. More precisely, choose an oriented orthonormal frame at $x \in N$ and use it to identify $T_{x} N$ with $\mathbf{R}^{2 n}$. This identification induces another identification $\pi_{2}^{-1}(x) \leftrightarrow \mathcal{F}=S O(2 n) / U(n)$. Thus $V_{(x, J)}$ is identified with the tangent space at some point of $S O(2 n) / U(n)$ and translating this tangent space to the identity coset of $S O(2 n) / U(n)$ we obtain the identification

$$
V_{(x, J)} \leftrightarrow \mathbf{n}=\mathbf{u}(n)^{\perp} \subset \mathbf{o}(2 n) .
$$

An invariant almost complex structure on $S O(2 n) / U(n)$ by restriction defines a complex structure on a vector space $n$, and hence one on $V_{(x, J)}$. Taking the direct sum of this "vertical" complex structure with the canonical complex structure defined on $H_{(x, J)}$ above at every $(x, J) \in \mathcal{T}$ we obtain an almost complex structure on $\mathcal{T}$. To put it another way, we obtain an almost complex structure on $\mathcal{T}$ by decreeing that the 1 -forms on $S O(N)$ given by

$$
\Phi^{i}=\Theta^{2 i-1}+i \Theta^{2 i}(1 \leqslant i \leqslant n), \quad \Theta^{\prime i j}(1 \leqslant i<j \leqslant n)
$$

pull back (by a local section) to give type $(1,0)$ forms on $T$. This almost complex structure will be denoted by

$$
J_{H} \oplus \sum \varepsilon_{i j} J_{1}
$$

Summing up, we have

PROPOSITION. There are $2^{\gamma}, \gamma=n(n-1) / 2$, many natural almost complex structures on $T \rightarrow N$, where $\operatorname{dim} N=2 n$.

Example. Let $N=S^{4}$. Then $S O(N)=S O(5)$ and

$$
\mathcal{T}=S O(5) / U(2) \stackrel{\cong}{\rightrightarrows} S p(2) / U(1) \times S p(1) \stackrel{\cong}{\rightarrow} \mathrm{C} P^{3} .
$$

The usual complex structure on $C P^{3}$ corresponds to $J_{H} \oplus \sum \varepsilon_{12} J_{1}$ with $\varepsilon_{12}=1$. 
Notation. $J_{+}=J_{h} \oplus \varepsilon_{i j} J_{1}$ with every $\varepsilon_{i j}=1 ; J_{-}=J_{h} \oplus \sum \varepsilon_{i j} J_{1}$ with every $\varepsilon_{i j}=-1$.

Remark. The almost complex structures $J_{+}$and $J_{-}$have been studied extensively by various authors; for example, see $[2,3,4,5,6,7]$. However, the remaining almost complex structures on $\mathcal{T}$, to the author's knowledge, have not been explored.

Proposition. With the possible exception of $J_{+}$the above almost complex structures on $\mathcal{T}$ are never integrable.

PRoOF: Exterior differentiate the forms $\Phi^{i}$ and $\Omega^{\prime i j}$.

\section{References}

[1] A. Borel and F. Hirzebruch, 'Characteristic classes and homogenous spaces I', Amer. J. Math. 80 (1958), 458-536.

[2] R.L. Bryant, 'Lie groups and twistor spaces', Duke Math. J. 52 (1985), 223-262.

[3] J. Eells and S. Salamon, 'Twistorial constructions of harmonic maps of surfaces into four-manifold', Ann. Scuola Norm. Sup. Pisa Cl. Sci. 12 (1985), 589-640.

[4] G.R. Jensen and M. Rigoli, 'Twistors and Gauss lifts of surfaces in four-manifolds', preprint.

[5] J. Rawnsley, 'Twistor methods', Lecture Notes in Math. 1283 (1987), 97-133.

[6] S. Salamon, 'Topics in four dimensional Riemannian geometry', Lecture Notes in Math. 1022 (1983), 34-124.

[7] S. Salamon, 'Harmonic and holomorphic maps', Lecture Notes in Math. 1164 (1985), 182-224.

[8] K. Yang, Almost Complex Homogeneous Spaces and Their Submanifolds (World Scientific Publishing, 1987).

Department of Mathematics,

Arkansas State University,

State University AR72467,

United States of America 